\title{
Neuroprotective Effects of Kangen-karyu on Spatial Memory Impairment in an 8-Arm Radial Maze and Neuronal Death in the Hippocampal CA1 Region Induced by Repeated Cerebral Ischemia in Rats
}

\author{
Fengling $\mathrm{Pu}^{1}$, Kyoko Motohashi ${ }^{1}$, Tomohiro Kaneko ${ }^{1}$, Yurika Tanaka ${ }^{1}$, Naomi Manome ${ }^{1}$, Keiichi Irie ${ }^{1,2}$, \\ Jirou Takata ${ }^{3}$, Nobuaki Egashira ${ }^{4}$, Ryozo Oishi ${ }^{4}$, Takuya Okamoto ${ }^{5}$, Yasuo Sei ${ }^{5}$, Takako Yokozawa ${ }^{6}$, \\ Kenichi Mishima ${ }^{1,2, *}$, Katsunori Iwasaki ${ }^{1,2}$, and Michihiro Fujiwara ${ }^{1}$ \\ ${ }^{I}$ Department of Neuropharmacology, ${ }^{3}$ Laboratory of Drug Design and Drug Delivery, Faculty of Pharmaceutical Sciences, \\ Fukuoka University, Fukuoka 814-0180, Japan \\ ${ }^{2}$ Advanced Materials Institute, Fukuoka University, Fukuoka 814-0180, Japan \\ ${ }^{4}$ Department of Pharmacy, Kyushu University Hospital, Fukuoka 812-8582, Japan \\ ${ }^{5}$ Iskra Industry Co., Ltd., Tokyo 103-0027, Japan \\ ${ }^{6}$ Institute of Natural Medicine, University of Toyama, Toyama 930-0194, Japan
}

Received September 4, 2008; Accepted January 15, 2009

\begin{abstract}
In the present study, we investigated the neuroprotective effects of Kangen-karyu $(\mathrm{KGK})$ in a repeated cerebral ischemia model $(2 \times 10 \mathrm{~min}, 1$-h interval). A 21 -day pre- and postischemic treatment with KGK $(10-300 \mathrm{mg} / \mathrm{kg})$ and aspirin $(5 \mathrm{mg} / \mathrm{kg})$ improved the spatial memory impairment and neuronal death in the hippocampal CA1 region induced by repeated cerebral ischemia. However, a 7-day post-ischemic treatment with KGK did not attenuate the spatial memory impairment and neuronal death in this model. To determine the mechanism of action of KGK, we investigated the effects of a 14-day pre-ischemic treatment with KGK on cerebral blood flow in the hippocampal area of the repeated cerebral ischemia model using laser Doppler flowmetry. The 14-day pre-ischemic treatment with KGK increased the cerebral blood flow during reperfusion. These results suggest that a 21-day pre- and post-ischemic treatment with KGK can protect against brain damage caused by cerebral ischemia by increasing the cerebral blood flow in the hippocampal area.
\end{abstract}

Keywords: Kangen-karyu, repeated cerebral ischemia, cerebral blood flow, neuronal death

\section{Introduction}

Kangen-karyu (KGK) was developed from the Chinese medicine Guanxin 2hao, which was originally used to treat coronary heart disease in China, and is composed of Salvia miltiorrhiza, Cnidii Rhizoma, Carthamus tinctorius L., Saussurea lappa CLARKE, Paeoniae Radix, and Cyperus rotundus L. In Japan, it is currently used to prevent lifestyle-related diseases, such as hypertension, dizziness, stiff neck, and heart palpitations. Several compounds of KGK are popular for

*Corresponding author. Kenichi@fukuoka-u.ac.jp Published online in J-STAGE on March 11, 2009 (in advance) doi: 10.1254 /jphs.08245FP improving cardiovascular events. For example, $S$. miltiorrhiza is a well-known traditional medicine for treating cardiovascular diseases in Asia, and its aqueous solution was reported to relax the rat coronary artery (1). C. tinctorius L. improves peripheral vascular disease as a Chinese traditional medicine. Hydroxysafflor yellow A, the main component of $C$. tinctorius L., was reported to reduce blood viscosity and thereby improve focal cerebral ischemia (2). Paeoniae Radix is a crude drug that was found to improve blood flow in Asia and Europe, and it was also reported to relax the isolated rat thoracic aorta (3). Cnidii Rhizoma was reported to decrease pig atrial contraction and slightly increase the beat rates of isolated atria (4). Taken together, these pieces of evidence suggest that KGK may increase 
cerebral blood flow.

The KGK extract has been reported to inhibit the generation of nitric oxide, superoxide, and hydroxyl radicals; enhance the activities of the antioxidative superoxide dismutase enzymes in hepatic tissues; and reduce the hepatic lipid peroxidation level that increased with aging in senescence-accelerated mice (5). KGK was also reported to increase cell viability and prolong lifespan in $\mathrm{H}_{2} \mathrm{O}_{2}$-induced human diploid fibroblasts (6). These results suggest that KGK can improve blood circulation and increase antioxidant activities, indicating that it may provide benefits for cerebral ischemia.

In the present study, we used a stroke model developed on the basis of the model generated by Pulsinelli and Brierley (7), which was designated the repeated cerebral ischemia model. In this model, we previously found a correlation between spatial memory impairment and hippocampal CA1 neuronal death, which were induced by two 10-min periods of cerebral ischemia with an interval of $1 \mathrm{~h}(8,9)$. Changes in the GluR2 (AMPAreceptor subunit) mRNA level and bcl family expression in the hippocampus following ischemia-reperfusion are thought to be related to the appearance of TUNELpositive cells in the CA1 region $(10,11)$. It is well known that cerebral blood flow exhibits a physiologic relationship with cerebral oxygen metabolism. In either global or focal cerebral ischemia, cerebral blood flow is significantly reduced in the brain regions that are supplied with oxygen by the occluded vessel. Choto-san, a traditional Chinese medicine, was reported to improve cerebral blood flow, prevent the occurrence of stroke, and prolong lifespan in stroke-prone spontaneously hypertensive rats $(12,13)$. We previously showed that nilvadipine, a calcium-channel blocker that increases cerebral blood flow (14), improved the spatial memory impairment and neuronal apoptosis in the repeated cerebral ischemia model (15). In the present study, we studied the effects of KGK on the spatial memory impairment and neuronal damage in the hippocampal CA1 region induced by repeated cerebral ischemia in rats. To investigate the mechanism of action of KGK, we also investigated its effects on cerebral blood flow in the hippocampal area in the repeated cerebral ischemia model.

\section{Materials and Methods}

\section{Materials}

KGK was supplied by ISKRA INDUSTRY CO., LTD. (Tokyo). The KGK extract used in this study comprised $4.5 \mathrm{~g}$ of Salvia miltiorrhizae Radix (root of $S$. miltiorrhiza BUNGE), $2.25 \mathrm{~g}$ of Cnidii Rhizoma (rhizome of Cnidium officinale MAKINO), $2.25 \mathrm{~g}$ of Carthami Flos (petal of C. tinctorius L.), $2.25 \mathrm{~g}$ of Paeoniae Radix (root of Paeonia lactiflora PALLAS), $1.125 \mathrm{~g}$ of S. lappa ClARKE root, and $1.125 \mathrm{~g}$ of Cyperi Rhizoma (rhizome of $C$. rotundus L.). These herbs were extracted with 25 volumes of water at $100^{\circ} \mathrm{C}$ for $1 \mathrm{~h}$. After filtration, the solution was evaporated under reduced pressure to give an extract with a $44 \%$ yield relative to the weight

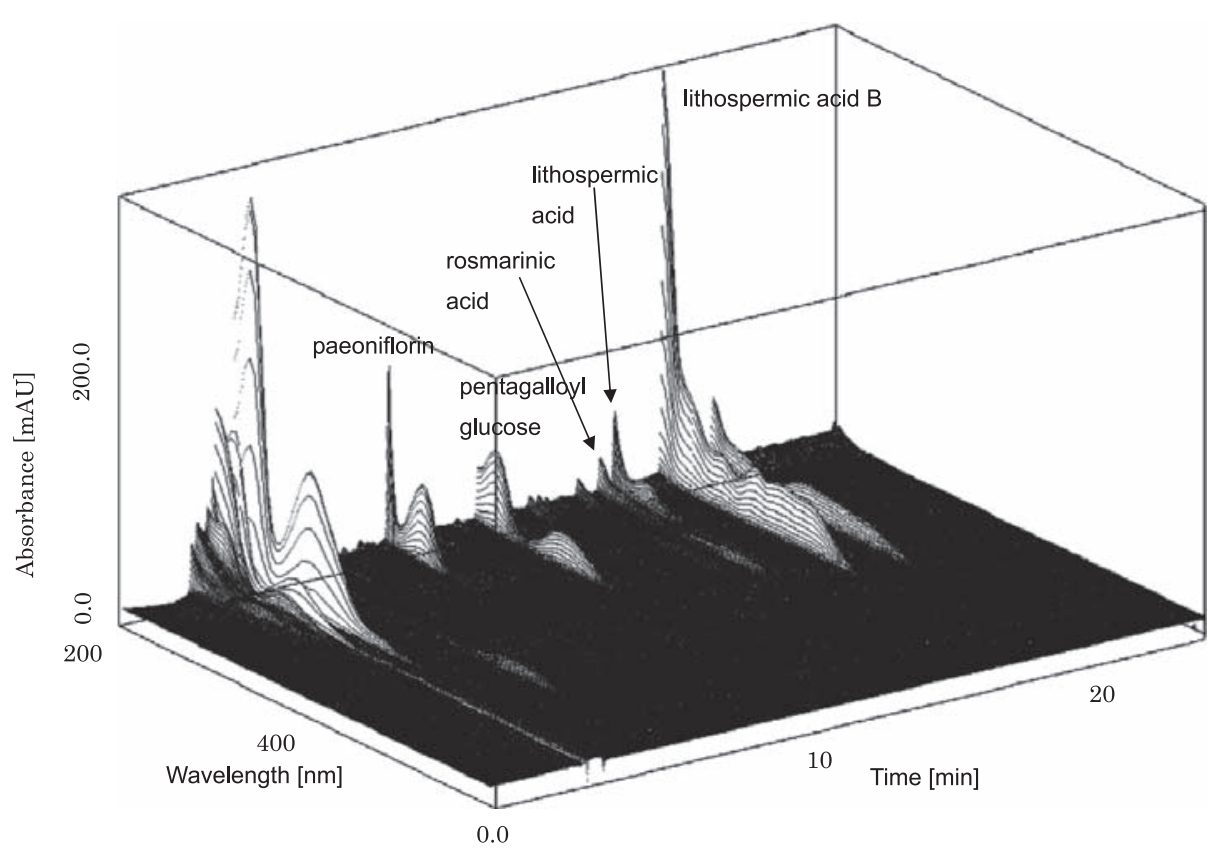

Fig. 1. Three-dimensional HPLC profile of the KGK extract. 
of the starting materials. A three-dimensional HPLC profile of the KGK extract used in the present study is shown in Fig. 1. Aspirin (Lot. EWL.3355) was purchased from Wako Pure Chemical Industries, Ltd. (Osaka).

\section{Animal and treatments}

Male Wistar rats weighing 250-300 g were obtained from Kyudo Co., Ltd. (Saga). They were housed in groups of 4 to 5 per cage in a temperature-controlled room $\left(23 \pm 2^{\circ} \mathrm{C}\right)$ with a relative humidity of $60 \pm 10 \%$ and maintained under a 12 -h light-dark cycle. All procedures regarding animal care and use were carried out based on the regulations dictated by the Experimental Animal Care and Use Committee of Fukuoka University. The rats were treated with KGK or aspirin that had been trained in an 8-arm radial maze task and required the spatial memory. For the 21-day pre- and postischemic treatment experiment, KGK or aspirin was orally administered once a day for 13 days. On day 14 , the administration was performed at $1 \mathrm{~h}$ before the first occlusion, and the administration was continued for a further 7 days. For the 7-day post-ischemic treatment experiment, rats were treated with KGK at $1 \mathrm{~h}$ after the first occlusion, and the administration was then continued for a further 6 days. The last administration in both experiments was performed at $1 \mathrm{~h}$ before retraining. Distilled water was administered to the control group.

\section{8-Arm radial maze task}

Behavioral testing using an 8-arm radial maze task (Neuroscience Co., Tokyo) was conducted as previously reported (16). The maze consisted of a central platform (24 cm in diameter) with 8 arms that extended radially. Rats were allowed to visit each arm to eat 8 pellets in food cups located near the end of each arm. Each test animal was trained once per day to memorize the apparatus. The performance of the test animals in each trial was assessed using three parameters: number of correct choices in the initial 8 chosen arms, number of errors (defined as choosing arms that had already been visited), and elapsed time before the animal ate all 8 pellets. When the test animals made 7 or 8 correct choices and no more than one error in three successive sessions, they were deemed to have memorized the maze. In other words, the rats had acquired spatial memory of the 8 -arm radial maze. The rats were then treated with KGK or underwent the cerebral ischemia treatment. The improvement of spatial memory impairment has been defined as when two parameters, correct choices and errors, were significantly affected simultaneously, that is, correct choices significantly increased and errors significantly decreased.

\section{Cerebral ischemia treatment}

Animals that had acquired spatial memory were subjected to cerebral ischemia according to our previous report (17). Briefly, rats were anesthetized with sodium pentobarbital $(50 \mathrm{mg} / \mathrm{kg}$, i.p.) and immobilized in a stereotaxic apparatus (Type SR-6; Narishige Scientific Instrument Laboratories, Tokyo). The bilateral arteries beneath the alar foramina of the first vertebra were electrocoagulated using a bipolar coagulator (MICRO3D; Mizuho Industrial Co., Tokyo), followed by bilateral exposure of both common carotid arteries by application of a hydraulic pressure vascular occluder (OC; diameter, $1.5 \mathrm{~mm}$; Technical Supply Co., Osaka) to each artery. On the following day, the common carotid arteries were compressed using the occluders operated by means of water-filled syringes, and cerebral circulation was interrupted for $10 \mathrm{~min}$. This was carried out twice at an interval of $1 \mathrm{~h}$. Rats that did not demonstrate loss of their righting reflex during arterial occlusion were excluded from subsequent experiments. Sham-operated control rats underwent cauterization of the vertebral arteries and were fitted with occluders.

\section{Histologic examination}

Rats were sacrificed after the behavioral test by deep anesthetization with pentobarbital $(50 \mathrm{mg} / \mathrm{kg}$, i.p.) and transcardial perfusion with cold heparinized saline, followed by perfusion of $4 \%$ paraformaldehyde. The brains were then removed and postfixed overnight in paraformaldehyde, before being dehydrated and embedded in paraffin. Representative coronal sections (5- $\mu$ m-thick), including the dorsal hippocampus, were obtained using a rotary microtome and stained with hematoxylin and eosin (HE). Microscopic examination of the neuronal density of the CA1 region was carried out to detect neuronal damage in the hippocampus.

\section{Measurement of cerebral blood flow}

Hippocampal blood flow was measured using laser Doppler flowmetry (TBF-LC1; Unique Medical, Tokyo). A laser Doppler flowmetry probe (needle type, ON97066; tip diameter, $500 \mu \mathrm{m})$ was inserted into the left dorsal hippocampus (A: -3.8, L: 2.0, V: 2.0). Hippocampal blood flow was expressed as a percentage relative to the mean baseline blood flow set as $100 \%$.

\section{Statistical analyses}

Results are expressed as means \pm S.E.M. Data from the behavioral tests and counts of CA1 pyramidal neurons were analyzed using the Mann-Whitney $U$ test. Blood flow data were evaluated by one-way ANOVA followed by Dunnett's post-hoc test. The criterion for statistical significance was considered to be $P<0.05$. 


\section{Results}

\section{Effects of $K G K$ on spatial memory impairment in the 8-arm maze}

As shown in Fig. 2, rats subjected to repeated cerebral ischemia (vehicle-treated group) exhibited a significantly decreased number of correct choices and increased number of errors (correct choices and errors, $P<0.001$ ). Low doses of aspirin are seen as an effective medical intervention for acute myocardial infarction and prevention of secondary stroke in the clinic (18). Therefore, aspirin was used as the positive control in this experiment. Pre- and post-ischemic treatment with KGK (10 $300 \mathrm{mg} / \mathrm{kg}$ for 21 days $)$ or aspirin $(5 \mathrm{mg} / \mathrm{kg})$ significantly decreased the spatial memory impairment induced by repeated ischemia during the 8 -arm radial maze test (correct choices and errors, $P<0.05, P<0.01$, and $P<0.001)$. In contrast, post-ischemic treatment with KGK $(100-300 \mathrm{mg} / \mathrm{kg}$ for 7 days) did not decrease the spatial memory impairment induced by repeated cerebral ischemia (Fig. 3).

\section{Effects of KGK on neuronal death in the hippocampal CAl area}

HE staining revealed pyknosis, eosinophilia, karyor-

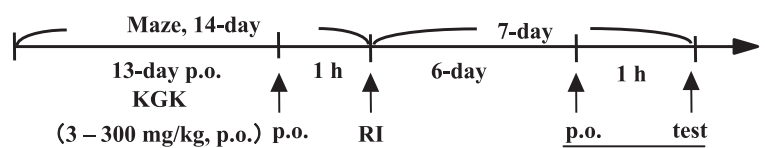

$\begin{array}{lll}(3-300 \mathrm{mg} / \mathrm{kg}, \text { p.o.) p.o. } & \text { RI } & \text { 7th day after RI } \\ \text { Aspirin }(5 \mathrm{mg} / \mathrm{kg} \text {, p.o.) } & \end{array}$

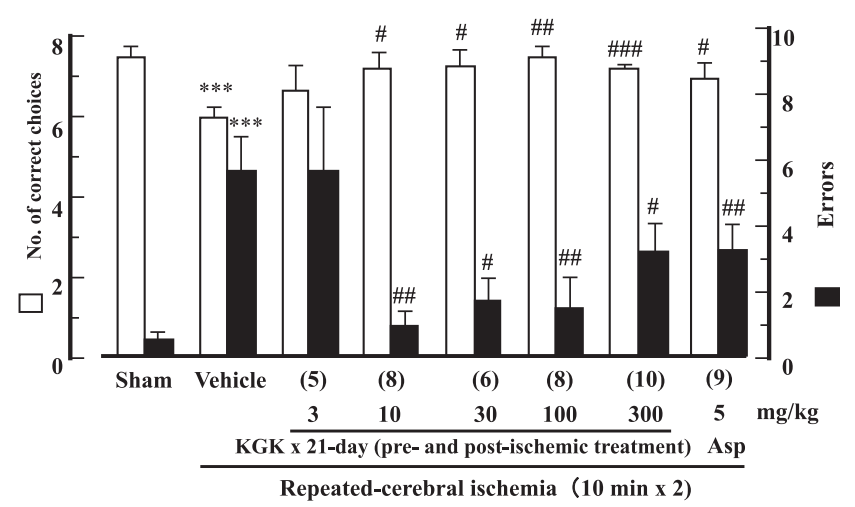

Fig. 2. Effects of a 21-day pre- and post-ischemic treatment with KGK or aspirin (Aps) on repeated cerebral ischemia-induced impairment of spatial memory in an 8-arm radial maze test in rats. Animals were administered various doses of KGK or Asp p.o. for 21 days. Before the repeated ischemia, KGK or Aps was administered for 13 days. On day 14, KGK or Asp was administered $1 \mathrm{~h}$ before the first occlusion and then administered for a further 7 days. RI in the schedule means repeated cerebral ischemia. Each filled column or empty column represents the mean value of the number of correct choices or errors, respectively $(\mathrm{n}=5-10)$. ${ }^{* * *} P<0.001$ vs. the sham group; ${ }^{\#} P<0.05,{ }^{\#} P<0.01,{ }^{\# \#} P<0.001$ vs. the vehicle group; MannWhitney $U$ test. rhexis, and chromosome condensation in the CA1 pyramidal neurons of the vehicle-treated repeated ischemia group compared to the sham group. Repeated cerebral ischemia induced cell death among the hippocampal CA1 pyramidal neurons $(P<0.001$, Fig. 4$)$. Pre-
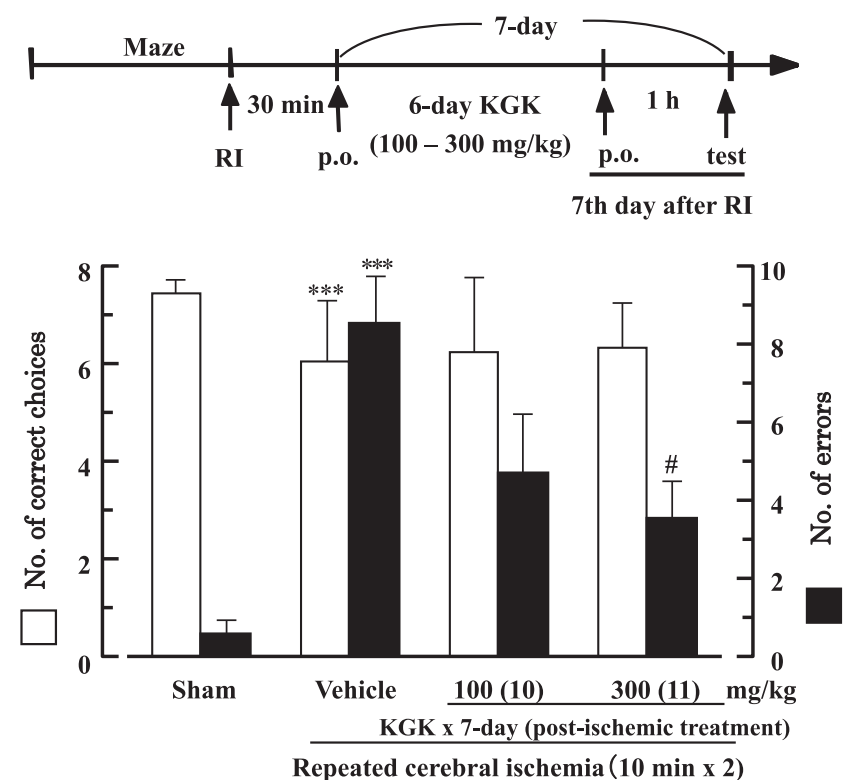

Fig. 3. Effects of a 7-day post-ischemic treatment with $\mathrm{KGK}$ and on repeated cerebral ischemia-induced impairment of spatial memory in an 8 -arm radial maze test in rats. Animals were administered various doses of KGK p.o. for 7 days after being subjected to ischemia. Each filled column or empty column represents the mean value of the number of correct choices or errors, respectively $(n=8-11)$. ${ }^{* * *} P<0.001$ vs. the sham group, ${ }^{*} P<0.05$ vs. the vehicle group, Mann-Whitney $U$ test.
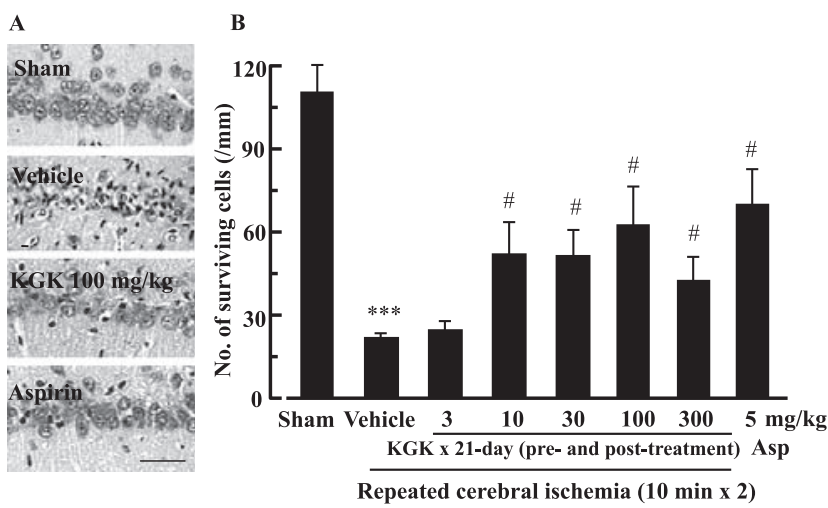

Fig. 4. Effects of a 21-day pre- and post-ischemic treatment with KGK or Asp on repeated cerebral ischemia-induced cell death in the hippocampal CA1 region. Images of surviving cells in the hippocampal CA1 region are shown in A. B shows the surviving cells in the CA1 region of the hippocampus expressed as the cell number $/ \mathrm{mm}$. Magnification: $\times 400(\mathrm{bar}=25 \mu \mathrm{m}) .{ }^{* * *} P<0.001$ vs. the sham group, ${ }^{\#} P<0.05$ vs. the vehicle group, Mann-Whitney $U$ test. The numbers of rats examined were $5-10$. 


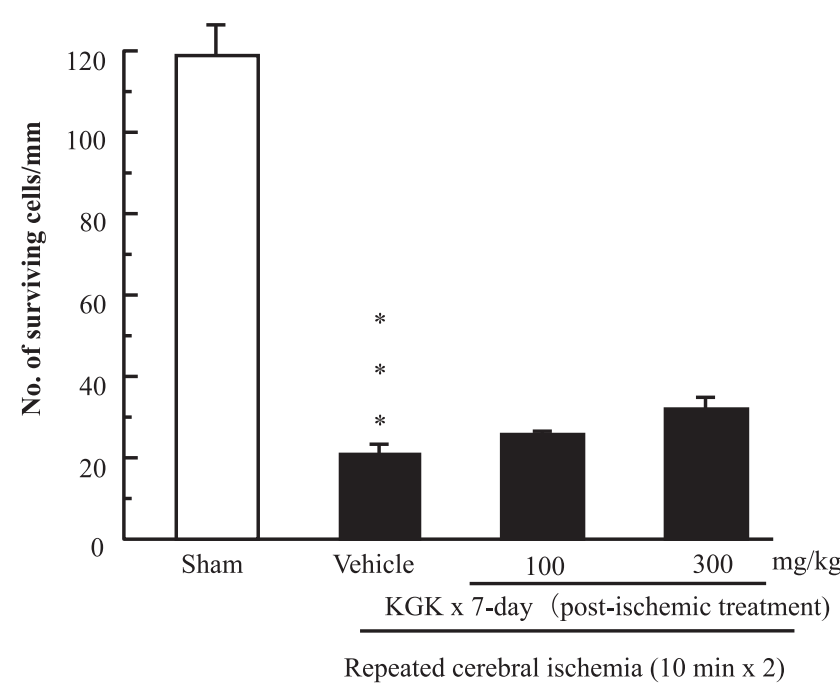

Fig. 5. Effects of a 7-day post-ischemic treatment with KGK on repeated cerebral ischemia-induced cell death in the hippocampal CA1 region. Surviving cells in the CA1 region of the hippocampus were expressed as the cell number $/ \mathrm{mm} .{ }^{* * *} P<0.001$ vs. the sham group (Mann-Whitney $U$ test). The numbers of rats examined were $8-11$.

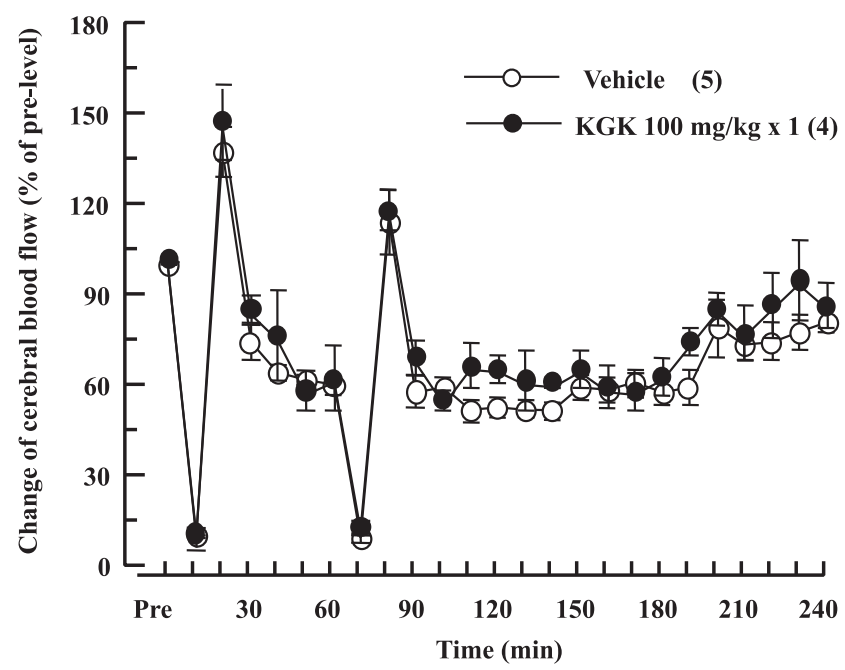

Fig. 6. Effects of a single treatment with KGK on hippocampal blood flow in rats subjected to repeated cerebral ischemia. In this experiment, KGK was administered $(100 \mathrm{mg} / \mathrm{kg}$, p.o.) once before the first occlusion. The mean value from -30 to 0 min was defined as $100 \%$, and the other values were expressed as percentages relative to the pre-ischemic level. The numbers of rats examined were $4-5$.

and post-ischemic treatment with KGK $(10-300 \mathrm{mg}$ $/ \mathrm{kg}$ for 21 days $)$ or aspirin $(5 \mathrm{mg} / \mathrm{kg})$ significantly suppressed the neuronal death $(P<0.05$, Fig. 4$)$, whereas the 7-day post-ischemic treatment with KGK failed to prevent cell death in this area (Fig. 5).

\section{Effects of KGK on cerebral blood flow}

A single treatment with KGK $(100 \mathrm{mg} / \mathrm{kg})$ did not

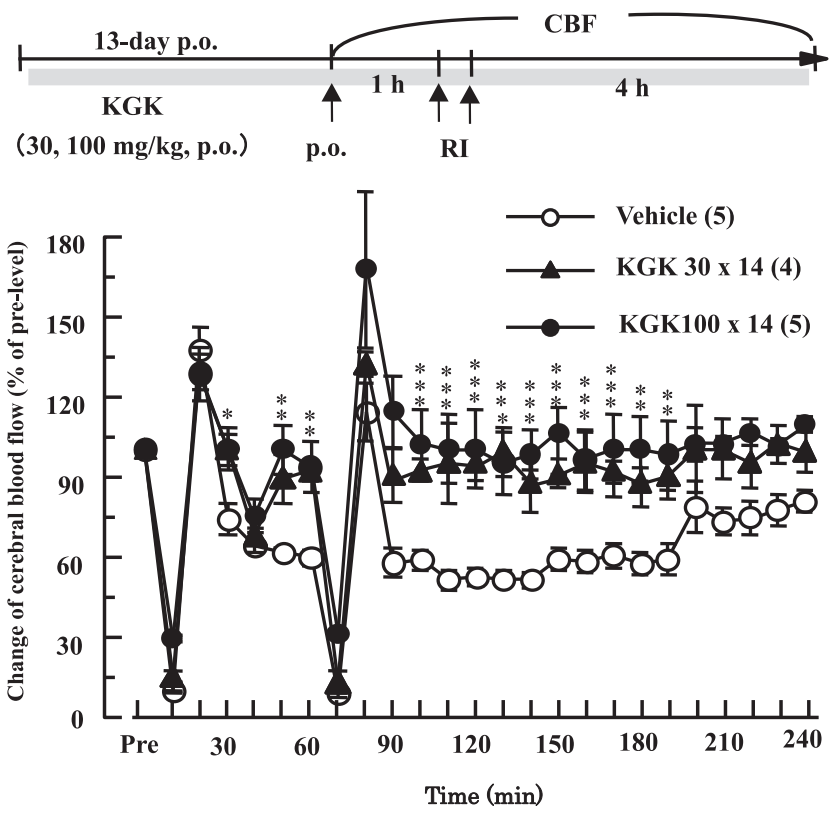

Fig. 7. Effects of a 14-day pre-ischemic treatment with KGK on hippocampal blood flow in rats subjected to repeated cerebral ischemia. In this experiment, KGK was administered for 13 days before ischemia. On day $14, \mathrm{KGK}$ was administered at $1 \mathrm{~h}$ before the first occlusion. The mean value from -30 to 0 min was defined as $100 \%$, and the other values were expressed as percentages relative to the pre-ischemic level. The number of rats examined was $4-5$. ${ }^{*} P<0.05,{ }^{*} P<0.01,{ }^{* * *} P<0.001$ vs. the sham group (Dunnett's post-hoc test).

affect hippocampal blood flow in the cerebral ischemia model (Fig. 6). In contrast, a 14-day pre-ischemic treatment with $\mathrm{KGK}(30,100 \mathrm{mg} / \mathrm{kg})$ significantly increased the blood flow in the hippocampal region after the first occlusion (Fig. 7).

\section{Discussion}

The present study has demonstrated that the 21-day pre- and post-ischemic treatment with KGK and aspirin significantly prevented the spatial memory impairment and neuronal death in the hippocampal CA1 area in rats subjected to repeated cerebral ischemia, whereas a 7-day post-ischemic treatment with KGK did not. Furthermore, the 14-day pre-ischemic treatment with KGK increased the blood flow in the hippocampal area during reperfusion. These results suggest that pre-ischemic administration of KGK prevents the spatial memory impairment and neuronal death induced by repeated cerebral ischemia by increasing the cerebral blood flow.

In the 8-arm radial maze (Fig. 2), the numbers of correct choices were the highest at a dose of $100 \mathrm{mg} / \mathrm{kg}$ of all doses used, although the numbers of error were the lowest at a dose of $10 \mathrm{mg} / \mathrm{kg}$. In addition, in the hippocampal CA1 region, the numbers of surviving cells 
were the highest at a dose of $100 \mathrm{mg} / \mathrm{kg}$. Therefore we examined the post-ischemic treatment of $100 \mathrm{mg} / \mathrm{kg}$ KGK on memory impairment, neuronal death, and cerebral blood flow. The 7-day post-ischemic treatment with KGK did not ameliorate either the spatial memory impairment or neuronal death in the hippocampal CA1 area. A single treatment with KGK did not change the blood flow in the hippocampal area during reperfusion in the cerebral blood flow experiments, but the 14-day treatment with KGK significantly increased the blood flow in the repeated cerebral ischemia model. It is thought that the neuroprotective mechanism of KGK is related to the ischemic cerebral blood flow. In fact, many papers reported that L-arginine, an NO donor, reduced the infarction area and elevated the functional recovery by increasing the cerebral blood flow $(19,20)$. A mild hypertension and blood flow increasing were reported to decrease the amount of cerebral ischemic injury after cerebral ischemia (21), and pharmacological blood pressure augmentation and blood flow restoration treatment were used for the acute phase treatment after ischemia in the clinic $(22,23)$. These results suggested that the neuroprotective mechanism of KGK was related to the ischemic cerebral blood flow.

Oxidative free radicals are known to increase during reperfusion after ischemia. The presence of oxidative free radicals during reperfusion after cerebral ischemia is regarded as one of the main risk factors for neuronal damage. It had been determined that KGK extracts protected against cellular senescence by inhibiting reactive oxygen species generation and regulating the antioxidantive status through 10 -week treatment $(100 \mathrm{mg}$ $/ \mathrm{kg}$ ) in senescence-accelerated mice (5) and inhibited lipid peroxidation against hypercholesterolemia (24). Many studies have reported that $S$. miltiorrhiza, $C$. tinctorius, and Paeoniae Radix can scavenge oxidative free radicals both in vivo and in vitro $(25-27)$. We further showed that S. miltiorrhiza, C. tinctorius, and Paeoniae Radix had strong free radical-scavenging activities for 1,1-diphenyl-2-picrylhydrazyl radicals in vitro (data not shown). Therefore, the neuroprotective mechanism of $\mathrm{KGK}$ in the repeated ischemia model may be related to its effects of increasing the cerebral blood flow and scavenging oxidative free radicals.

As described above, KGK is comprised of six herbs at ratios of $4: 2: 2: 2: 1: 1$ and the largest component is $S$. miltiorrhiza. According to traditional Chinese medicine theory, S. miltiorrhiza is considered to be the most important component of KGK. Cryptotanshinone, a component of $S$. miltiorrhiza, has been reported to induce a concentration-dependent increase in endothelial nitric oxide synthase (eNOS) expression without significantly changing neuronal nitric oxide synthase
(nNOS) expression in human umbilical vein endothelial cells (28). Cryptotanshinone and tanshinone IIA from S. miltiorrhiza were shown to have dilatory effects on the rat coronary artery (29). Tanshinone IIA was also found to suppress human aortic smooth muscle cell migration and matrix metalloproteinase- 9 activity by inhibiting the activities of the PI-3K/AKT and ERK1/2 signal pathways (30). A $P$. lactiflora extract was reported to relax prostaglandin F $\alpha$-precontracted aortic ring preparations from isolated rat aorta with the endothelium (31). It was also reported that ligustilide, cnidilide, and senkyunolide from C. officinale MAKINO can relax the centrally acting muscle in the crossed extensor reflex in anesthetized rats (32). Taking all the observations together, the neuroprotective mechanism of KGK is considered to involve an increase in the cerebral blood flow through the vasorelaxation effect and scavenging of oxidative free radicals during reperfusion in the repeated cerebral ischemia model.

In conclusion, we have shown that a 21-day pre- and post-ischemic treatment with KGK and aspirin decreased the spatial memory impairment and neuronal death in the hippocampal CA1 area induced by repeated cerebral ischemia in rats. This neuroprotective mechanism of KGK may be related to an increase in the blood flow in the hippocampal area.

\section{References}

1 Lam FF, Yeung JH, Chan KM, Or PM. Relaxant effects of danshen aqueous extract and its constituent danshensu on rat coronary artery are mediated by inhibition of calcium channels. Vascul Pharmacol. 2007;46:271-277.

2 Yoo MY, Lee BH, Choi YH, Lee JW, Seo JH, Oh KS, et al. Vasorelaxant effect of the rootbark extract of Paeonia moutan on isolated rat thoracic aorta. Planta Med. 2006;72:1338-1341.

3 Zhu HB, Zhang L, Wang ZH, Tian JW, Fu FH, Liu K, et al. Therapeutic effects of hydroxysafflor yellow A on focal cerebral ischemic injury in rats and its primary mechanisms. J Asian Nat Prod Res. 2005;7:607-613.

4 Nakazawa K, Fujimori K, Inoue K, Sekita S, Takanaka A. Effects of extract from a herbal drug, cnidium rhizome (senkyu), on contraction, heart rates and membrane potentials of isolated guinea pig atria. Yakugaku Zasshi. 1989;109:662-671.

5 Satoh A, Yokozawa T, Cho EJ, Okamoto T, Sei Y. Antioxidative effects related to the potential anti-aging properties of the Chinese prescription Kangen-karyu and Carthami Flos in senescence-accelerated mice. Arch Gerontol Geriatr. 2004;39: 69-82.

6 Yokozawa T, Cho EJ, Okamoto T, Sei Y. Effects of the Chinese prescription Kangen-karyu and its crude drug Tanjin on ageing process in rats. J Pharm Pharmacol. 2006;58:1591-1599.

7 Pulsinelli WA, Brierley JB. A new model of bilateral hemispheric ischemia in the unanesthetized rat. Stroke. 1979;10:267272.

8 Chung E, Iwasaki K, Mishima K, Egashira N, Fujiwara M. 
Repeated cerebral ischemia induced hippocampal cell death and impairments of spatial cognition in the rat. Life Sci. 2002;72: 609-619.

9 Iwasaki K, Chung E, Egashira N, Hatip-Al-Khatib I, Mishima K, Egawa $\mathrm{T}$, et al. Non-NMDA mechanism in the inhibition of cellular apoptosis and memory impairment induced by repeated ischemia in rats. Brain Res. 2004;995:131-139.

10 Hatip-Al-Khatib I, Iwasaki K, Egashira N, Ishibashi D, Mishima K, Fujiwara M. Comparison of single- and repeated-ischemiainduced changes in expression of flip and flop splice variants of AMPA receptor subtypes GluR1 and GluR2 in the rats hippocampus CA1 subregion. J Pharmacol Sci. 2007;103:83-91.

11 Irie K, Mishima K, Ishibashi D, Egashira N, Iwasaki K, Fujiwara $\mathrm{M}$. Involvement of bcl-family expression in the spatial memory impairment induced by repeated ischemia. Life Sci. 2002;72: 621-629.

12 Sugimoto A, Goto K, Ishige A, Komatsu Y, Miyamoto KI. Effect of Choto-san, a Kampo medicine, on the cerebral blood flow autoregulation in spontaneously hypertensive rats. Jpn J Pharmacol. 2000;83:135-142.

13 Shimada Y, Yang Q, Yokoyama K, Goto H, Kasahara Y, Sekiya $\mathrm{N}$, et al. Choto-san prevents occurrence of stroke and prolongs life span in stroke-prone spontaneously hypertensive rats. Am J Chin Med. 2003;31:79-85.

14 Tanaka R, Miyasaka Y, Maruyama S, Nagai S, Fujii K. Effects of nilvadipine (a dihydropyridine-type calcium entry blocker) on cerebral blood flow in acute experimental brain ischemia in rats. Neurol Res. 1996;18:325-328.

15 Iwasaki K, Mishima K, Egashira N, Al-Khatib IH, Ishibashi D, Irie $\mathrm{K}$, et al. Effect of nilvadipine on the cerebral ischemiainduced impairment of spatial memory and hippocampal apoptosis in rats. J Pharmacol Sci. 2003;93:188-196.

16 Pu F, Mishima K, Egashira N, Iwasaki K, Kaneko T, Uchida T, et al. Protective effect of buckwheat polyphenols against longlasting impairment of spatial memory associated with hippocampal neuronal damage in rats subjected to repeated cerebral ischemia. J Pharmacol Sci. 2004;94:393-402.

17 Pu F, Mishima K, Irie K, Motohashi K, Tanaka Y, Orito K, et al. Neuroprotective effects of quercetin and rutin on spatial memory impairment in an 8-arm radial maze task and neuronal death induced by repeated cerebral ischemia in rats. J Pharmacol Sci. 2007;104:329-334.

18 Kirshner HS. Prevention of secondary stroke and transient ischaemic attack with antiplatelet therapy: the role of the primary care physician [corrected]. Int J Clin Pract. 2007;61:17391748.

19 Zhang F, White JG, Iadecola C. Nitric oxide donors increase blood flow and reduce brain damage in focal ischemia: evidence that nitric oxide is beneficial in the early stages of cerebral ischemia. J Cereb Blood Flow Metab. 1994;14:217-226.

20 Morikawa E, Moskowitz MA, Huang Z, Yoshida T, Irikura K,
Dalkara T. L-arginine infusion promotes nitric oxide-dependent vasodilation, increases regional cerebral blood flow, and reduces infarction volume in the rat. Stroke. 1994;25:429-435.

21 Wityk RJ, Lewin JJ 3rd. Blood pressure management during acute ischaemic stroke. Expert Opin Pharmacother. 2006; 7:247258.

22 Hosomi N, Mizushige K, Kitadai M, Ohyama H, Ichihara SI, Takahashi $\mathrm{T}$, et al. Induced hypertension treatment to improve cerebral ischemic injury after transient forebrain ischemia. Brain Res. 1999;835:188-196.

23 Diringer MN, Axelrod Y. Hemodynamic manipulation in the neuro-intensive care unit: cerebral perfusion pressure therapy in head injury and hemodynamic augmentation for cerebral vasospasm. Curr Opin Crit Care. 2007;13:156-162.

24 Yokozawa T, Cho EJ, Sasaki S, Satoh A, Okamoto T, Sei Y. The protective role of Chinese prescription Kangen-karyu extract on diet-induced hypercholesterolemia in rats. Biol Pharm Bull. 2006;4:760-765.

25 Zhao GR, Zhang HM, Ye TX, Xiang ZJ, Yuan YJ, Guo ZX, et al. Characterization of the radical scavenging and antioxidant activities of danshensu and salvianolic acid B. Food Chem Toxicol. 2008;46:73-81.

26 Lee SC, Kwon YS, Son KH, Kim HP, Heo MY. Antioxidative constituents from Paeonia lactiflora. Arch Pharm Res. 2005; 28:775-783.

27 Koyama N, Kuribayashi K, Seki T, Kobayashi K, Furuhata Y, Suzuki K, et al. Serotonin derivatives, major safflower (Carthamus tinctorius L.) seed antioxidants, inhibit low-density lipoprotein (LDL) oxidation and atherosclerosis in apolipoprotein E-deficient mice. J Agric Food Chem. 2006;54:4970 4976.

28 Zhou Z, Wang SQ, Liu Y, Miao AD. Cryptotanshinone inhibits endothelin-1 expression and stimulates nitric oxide production in human vascular endothelial cells. Biochim Biophys Acta. 2006; 1760:1-9.

29 Lam FF, Yeung JH, Chan KM, Or PM. Mechanisms of the dilator action of cryptotanshinone on rat coronary artery. Eur J Pharmacol. 2008;578:253-260.

30 Jin UH, Suh SJ, Chang HW, Son JK, Lee SH, Son KH, et al. Tanshinone IIA from Salvia miltiorrhiza BUNGE inhibits human aortic smooth muscle cell migration and MMP-9 activity through AKT signaling pathway. J Cell Biochem. 2008;104:1526.

31 Goto H, Shimada Y, Akechi Y, Kohta K, Hattori M, Terasawa $\mathrm{K}$. Endothelium-dependent vasodilator effect of extract prepared from the roots of Paeonia lactiflora on isolated rat aorta. Planta Med. 1996;62:436-439.

32 Ozaki Y, Sekita S, Harada M. [Centrally acting muscle relaxant effect of phthalides (ligustilide, cnidilide and senkyunolide) obtained from Cnidium officinale Makino.] Yakugaku Zasshi. 1989;109:402-406. (text in Japanese with English abstract) 